Le traitement a été interrompu pour cause de toxicité chez 12 patients (7 \%) du bras Dasa, principalement pour toxicité hématologique de grade 3-4 (six patients) et épanchement pleural (cinq patients) et chez quatre (5\%) du bras IM, pour causes non hématologiques. La toxicité hématologique était comparable dans les deux bras : neutropénie de grade $3 / 4$ chez $12 \%$ des patients du bras Dasa et chez $16 \%$ du bras IM, et chez $29 \%$ des patients secondairement sous Dasa. Des complications cardiovasculaires ont été rapportées chez deux patients du bras Dasa et chez un patient du bras IM switchés vers le Dasa.

Les évènements indésirables graves non hématologiques les plus fréquents étaient des maux de tête dans le bras Dasa et une hypophosphatémie dans le groupe IM.

Bien que les résultats de cette étude montrent que l'obtention d'une RMM après 12 mois de traitement est supérieure dans le bras Dasa (29 versus $13 \%, \mathrm{p}=0,005$ ) et à 24 mois (64 versus $41 \%$ ), les taux de SSP et de SG ne sont pas significativement différents. Des études ont montré que l'obtention d'une réponse moléculaire majeure ou d'une réponse cytogénétique complètes était favorisée avec les ITK de deuxième génération mais il est difficile de montrer qu'un switch précoce pour des patients en réponse non optimale à trois mois donne une meilleure survie à long terme. Les résultats de cette étude demandent une réévaluation à long terme. Il est à noter que de nombreux effets secondaires sont survenus, dont certains ont eu pour conséquence des interruptions de traitement. Les cas d'épanchement pleuraux sous Dasa sont cependant rapportés en moins grand nombre (9\%) dans cette étude que dans les précédentes études d'enregistrement (14\%), peut-être du fait de la sélection de patients plus jeunes dans cette étude [3]. Au total, les facteurs de réponse optimale au traitement par ITK ne sont pas tous bien compris en dehors de l'apparition de mutations de résistance (tolérance, métabolisme, observance, immunologiques, etc.) et nécessitent d'être étudiés afin d'adapter au mieux le traitement à chaque patient. ]

\section{Références}

[1] Jain P, Kantarjian H, Nazha A, et al. Early responses predict better outcomes in patients with newly diagnosed chronic myeloid leukemia results with four tyrosine kinase inhibitor modalities. Blood 2013 ; 121 : 4867-74.

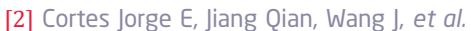
Dasatinib vs. imatinib in patients with chronic myeloid leukemia in chronic phase (CML-CP) who have not achieved an optimal response to 3 months of imatinib therapy : the DASCERN randomized study. Leukemia 2020 ; 34 : 2064-73.

[3] Kantarjian HM, Shah NP, Cortes JE, et al. Dasatinib or imatinib in newly diagnosed chronic phase chronic myeloid leukemia : 2-year follow-up from a randomized phase 3 trial (DASISION). Blood 2012 ; 119 : 1123-9.

\title{
Prise en charge de la splénomégalie chez les patients atteints de myélofibrose : quelles options en 2020 ?
}

\author{
Laly Nsiala \\ Jose Miguel Torregrosa Diaz \\ améliorer la qualité de vie de ces \\ patients.
}

a myélofibrose (MF) est un syndrome myéloprolifératif, caractérisée par une fibrose médullaire avec risque d'hématopoïèse extramédullaire au cours de l'évolution. Un des symptômes les plus fréquents est la splénomégalie (SMG), avec $38 \%$ des patients présentant une rate $>10 \mathrm{~cm}$ de débord costal. Elle est responsable de cytopénies et d'absence de rendement transfusionnel en lien avec une séquestration splénique, mais aussi de douleurs de l'hypocondre gauche, d'une perte de poids par satiété précoce en lien avec une compression gastrique et d'une hypertension portale. La diminution de la taille de la rate est donc un enjeu majeur pour

\section{Traitements validés dans la myélofibrose}

Les traitements classiquement essayés pour la SMG comprennent (figure 1) :

- l'hydroxyurée, efficace dans $\sim 40 \%$ de patients selon les séries, mais avec une toxicité hématologique limitante,

- l'interféron $\alpha$ (IFN $\alpha$ ), sans efficacité dans ce contexte,

- le melphalan à faibles doses, qui comporte surtout un risque élevé de néoplasies secondaires, pour une efficacité médiocre,

- les médicaments immunomodulateurs (thalidomide et lénalidomide), qui n'ont pas montré d'efficacité sur la SMG mais surtout sur l'anémie dans un sous-groupe de patients.

\section{Ruxolinitib}

Le ruxolitinib est un inhibiteur de la Janus kinase 2 (JAK2) indiqué dans le traitement des MF de risque intermédiaire et élevé suite aux résultats des études de phase III COMFORT [2]. Dans COMFORT I, qui comparait l'efficacité du ruxolinitib contre placebo, 41,9\% des patients traités par ruxolitinib ont obtenu une réduction du volume de la rate (RVR) $\geq 35 \%$ (versus $0,7 \%$ ). Des résultats similaires ont été obtenus dans COMFORT II (ruxolitinib versus meilleur traitement disponible), avec $53,4 \%$ des patients traités 


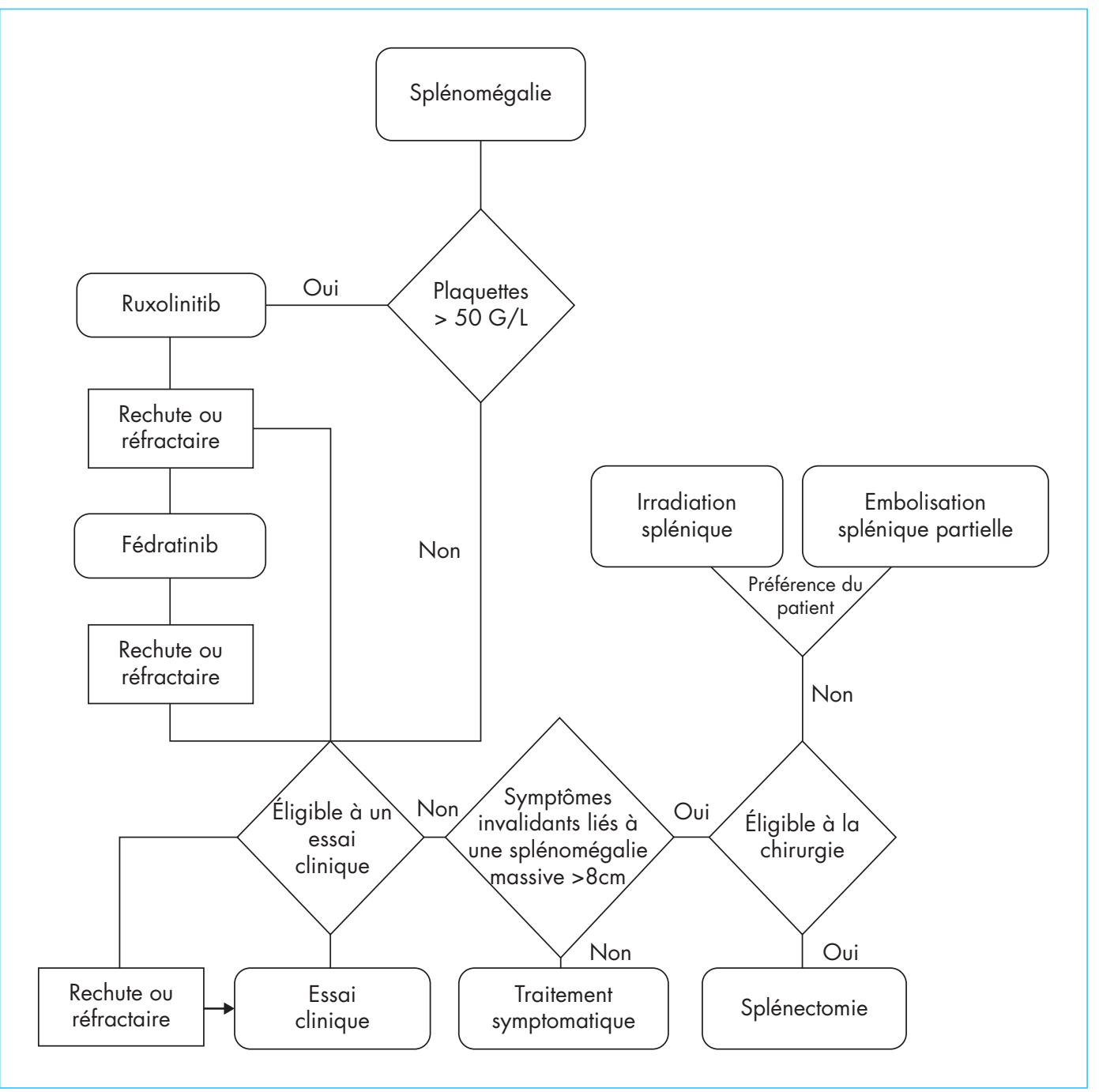

Algorithme de prise en charge de la splénomégalie (modifié d'après Tremblay et al., [1]).

par ruxolitinib ayant obtenu une RVR $\geq 35 \%$ (versus $0 \%$ ). Cependant $50 \%$ des patients ont arrêté leur traitement à trois ans, soit pour intolérance, soit en raison d'une progression de la maladie. Le pronostic des patients ayant arrêté le ruxolitinib étant sombre (survie globale de 14 mois), d'autres thérapeutiques sont nécessaires.

\section{Fédratinib}

Le fédratinib est un nouvel inhibiteur de JAK2 ayant une indication chez les patients non éligibles ou réfractaires au ruxolinitib. Dans JAKARTA-I [3] qui comparait l'efficacité du fédratinib en première ligne, à la posologie de 400 ou de $500 \mathrm{mg} / \mathrm{j}$ contre placebo, 36 et $40 \%$ des patients respectivement ont obtenu une RVR > $35 \%$ (versus $1 \%$ ). La limite principale du fédratinib et du ruxolinitib reste la toxicité hématologique, en particulier la thrombopénie, qui limite son utilisation, et une attention particulière à donner au risque d'encéphalopathie de Wernicke.
Nouvelles options thérapeutiques à l'étude

Nouveaux inhibiteurs de JAK2 De nouveaux agents inhibiteurs de JAK2 sont en cours d'étude : le momélotinib, un inhibiteur de JAK2, de JAK1 et d'ACVR1 et le pacritinib, un inhibiteur de JAK2 et de FLT3.

Dans l'étude de phase III SIMPLIFYI, 432 patients naïs au traitement ont été inclus pour recevoir soit le ruxolinitib soit le momélotinib. À 
deux ans de traitement, il n'a pas été démontré de différence significative en termes de RVR dans les deux groupes (29 versus 26,5\%). Des résultats similaires ont été obtenus dans SIMPLIFY-II, dans laquelle il n'y avait pas d'avantage significatif à l'utilisation de momélotinib par rapport à la meilleure thérapie disponible (7 versus $6 \%$ ). Toutefois, si le bénéfice semble limité pour ce qui concerne la splénomégalie, il semble plus important sur l'anémie, du fait de ses propriétés proérythropoïétiques, et son évaluation est en cours dans cette indication dans l'étude de phase III MOMENTUM (NCT04173494).

Dans les essais PERSIST [4], 18 à $19 \%$ des patients traités par pacritinib ont obtenu une RVR > $35 \%$, avec une efficacité et une tolérance correcte y compris chez des patients très thrombopéniques. La posologie de $200 \mathrm{mg}$ deux fois par jour semble être la plus efficace et a été retenue dans l'étude PACIFICA (NCT03165734) pour évaluer son profil d'efficacité et de tolérance chez un patient avec un taux de plaquettes $<50 \mathrm{G} / \mathrm{L}$.

\section{CPI-0610}

Le CPI-0610 est un agent inhibiteur des protéines BET (pour bromodomain and extraterminal) dont l'efficacité a été évaluée dans la MF. Dans une étude de phase II incluant des patients ayant reçu ou non du ruxolinitib, $94 \%$ des patients ayant reçu le CPI0610 en association au ruxolinitib ont obtenu une RVR significative, d'environ $17 \%$. Son efficacité semble tout aussi importante en monothérapie chez des patients n'ayant jamais reçu de ruxolinitib, avec $80 \%$ des patients ayant atteint l'objectif de RVR $>35 \%$. L'étude en double aveugle MANIFEST-2 (NCT02158858) cherche actuellement à évaluer son efficacité en première ligne, versus ruxolitinib, chez des patients naïs aux inhibiteurs de JAK2.

\section{Navitoclax}

Le navitoclax est un inhibiteur de BCL2/BCL-xL dont l'efficacité, en association au ruxolitinib, a été évaluée chez les patients ayant une réponse insuffisante sous ruxolitinib en monothérapie. Dans une étude incluant 24 patients, $29 \%$ ont obtenu une RVR $\geq 35 \%$ à deux ans et $42 \%$ au total au cours de l'étude.

D'autres molécules sont en cours d'évaluation en association au ruxolitinib tel que la thalidomide ou l'interféron pour tenter d'améliorer la profondeur et la durée de la réponse.

\section{Traitement palliatif}

\section{Splénectomie}

Aujourd'hui, la splénectomie (par laparoscopie dans la mesure du possible) garde sa place dans la prise en charge des patients non éligibles et/ou réfractaires aux inhibiteurs de JAK2 et non incluables dans un essai clinique, en cas de SPM volumineuse et symptomatique. Dans une étude incluant 314 patients atteints de MF, $77,6 \%$ ont obtenu une amélioration de leur symptomatologie après splénectomie avec une survie globale de 19 mois en moyenne. La sélection des patients doit cependant être la plus restrictive possible pour limiter les risques de complications péri- et postopératoires (évaluation des comorbidités et du taux de plaquettes, vaccination contre les germes encapsulés). Si le patient y est éligible, un traitement par inhibiteur de JAK2 devrait être repris en postopératoire pour limiter les risques d'hématopoï̀se extramédullaire hépatique.

\section{Irradiation splénique}

Pour les patients non éligibles à la chirurgie, l'irradiation splénique peut être une option, du fait de sa radiosensibilité. Le schéma le plus classique est une dose d'irradiation de 10 Gy délivrée en 10 fractions. Dans une étude menée chez 23 patients avant l'ère du ruxolitinib, $93 \%$ ont obtenu une RVR significative, pour une médiane de durée de réponse de six mois. Son intérêt peut aussi être son utilisation en prétransplantation pour améliorer la prise de greffe. Les principales complications sont l'apparition de cytopénies sévères et prolongées avec un retentissement clinique (aplasie fébrile, hémorragies) et des complications digestives (nausées, diarrhées) liées au champ d'irradiation.

\section{Embolisation splénique}

L'embolisation splénique partielle, sous couvert d'une antibioprophylaxie, peut aussi permettre d'améliorer la symptomatologie, tout en conservant une partie de rate fonctionnelle. Quelques cas ont été décrits dans les MF avec un effet sur l'augmentation du taux de plaquettes. La principale complication est la survenue de douleurs intenses survenant chez $75 \%$ des patients et pouvant nécessiter une hospitalisation pour contrôle de la douleur.

En conclusion, bien que les inhibiteurs de JAK2 aient radicalement amélioré la qualité de vie des patients, une majorité finira par $y$ être réfractaire, d'une part, et d'autre part, ces agents n'agissent pas sur la physiopathologie de la MF ni sur son évolution naturelle. L'utilisation de bithérapies et l'émergence de nouvelles thérapeutiques sont des pistes intéressantes pour améliorer aussi bien la qualité de vie que la survie globale. ]

\section{Références}

[1] Tremblay D, Schwartz M, Bakst R, et al. Modern management of splenomegaly in patients with myelofibrosis. Ann Hematol 2020 ; 99 (7) : 1441-51.

[2] Verstovsek S, Mesa RA, Gotlib J, et al. A double-blind, placebo- controlled trial of ruxolitinib 
for myelofibrosis. N Engl J Med 2012 ; 366 (9) : 799-807.

[3] Pardanani A, Harrison C, Cortes JE, et al. Safety and efficacy of fédratinib in patients with primary cal trial. JAMA Oncol 2015 ; 1 (5) : 643-51.

[4] Mesa RA, Vannucchi AM, Mead A, et al. Pacritinib versus best available therapy for the treat- ment of myelofibrosis irrespective of baseline cytopenias (PERSIST-1): an international, randomised, phase 3 trial. Lancet Haematol 2017 ; 4 (5) e225-36

\section{Adjonction du daratumumab à l'association bortézomib, lénalidomide et dexaméthasone dans le myélome multiple éligible à une autogreffe de cellules souches hématopoiétiques}

\section{Nicolas Stocker}

'association du bortézomib, du lénalidomide et de la dexaméthasone (VRD) est un standard de traitement de première ligne des patients atteints de myélome multiple nouvellement diagnostiqués (MMND) et éligibles à une autogreffe de cellules souches hématopoïétiques, permettant d'obtenir une médiane de survie sans progression de 50 mois et un taux de survie globale de $81 \%$ à quatre ans [1]. Récemment, l'étude CASSIOPEIA a démontré que l'adjonction du daratumumab (Dara), un anticorps monoclonal anti-CD38, au traitement d'induction associant $\mathrm{du}$ bortézomib, de la thalidomide et de la dexaméthasone améliorait significativement son efficacité chez ces patients [2]. Les auteurs de cette étude de phase II ont donc évalué l'adjonction du Dara au VRD dans ce contexte clinique [3].

Entre décembre 2016 et avril 2018, 207 patients ont été inclus dans cette étude multicentrique de phase II ayant comparé le VRD au Dara-VRD (104 patients dans le groupe DaraVRD, 103 patients dans le groupe VRD). Le critère de jugement principal était le taux de réponse complète (RC) stringente au terme de la consolidation. Les patients recevaient quatre cycles d'induction de 21 jours, une intensification thérapeutique par melphalan $\left(200 \mathrm{mg} / \mathrm{m}^{2}\right)$ suivie d'une autogreffe de cellules souches hématopoïétiques, puis deux cycles de consolidation de 21 jours chacun. Les groupes de traitement étaient équilibrés avec une médiane d'âge de 60 ans (29-70) et un délai médian depuis le diagnostic de 0,8 mois (0-61). Près de la moitié des patients présentaient un stade ISS (pour international staging system) I (48\%) et $16 \%$ des patients $(\mathrm{n}=30)$ présentaient un myélome multiple de haut risque cytogénétique (délétion du $17 \mathrm{p}$, translocations $[4,14]$ ou $[14,16])$.

Au terme des cycles de consolidation, le taux de RC dans le groupe Dara-VRD était supérieur à celui du groupe VRD (42 versus $32 \%$, OR : 1,57 ; 95\%CI : $0,87-2,82 ; \mathrm{P}=0,068$ avec un risque $\alpha$ de 0,10$)$. Les taux de réponse globale et de réponse de très bonne qualité à ce stade étaient significativement supérieurs dans le groupe Dara-VRD (99 versus $92 \% ; \mathrm{P}=0,016$ et 91 versus $73 \% ; \mathrm{P}=0,001)$. Avec un suivi médian de 22 mois, ces résultats étaient conservés, tant en termes de taux de RC (65 versus $45 \%$, OR : 1,98; 95\%CI : 1,12-3,49; $\mathrm{P}=0,01$ ) qu'en termes de taux de RC stringente (80 versus $61 \%$, OR 2,53 ; 95\%CI : 1,33$4,81 ; \mathrm{P}=0,005)$. Les taux de maladie résiduelle (MRD) indétectable (MRD : $10^{-5}$ ) dans le groupe Dara-VRD étaient significativement supérieurs à ceux du groupe VRD (respectivement 51 versus $20 \%$, P $<0,0001)$. En revanche, les taux de RC stringente et de MRD indétectable n'étaient pas différents dans les sous-groupes de patients présentant un score ISS à 3 et/ou un haut risque cytogénétique.

Sur le plan de la tolérance, les principaux effets indésirables de grade 3-4 dans les groupes DaraVRD et VRD étaient des neutropénies (41 versus $22 \%$ ), des lymphopénies (23 versus $22 \%$ ), des thrombopénies (16 versus $9 \%$ ), des leucopénies
(16 versus $9 \%$ ), et des pneumopathies (8 versus $11 \%$ ). L'incidence des infections de grade 3-4 était comparables entre les groupes (23 versus $22 \%$ ) et ces dernières engendraient une interruption de traitement chez respectivement 2 et $3 \%$ des patients. Quarante-deux pour cent (42\%) des patients du groupe Dara-VRD présentaient une réaction à l'infusion du Dara (seulement $6 \%$ de réaction de grade III, aucune de grade 4-5). Ces réactions concernaient majoritairement la première administration du traitement.

En conclusion, l'association DaraVRD permet un gain significatif en profondeur de réponse lors du traitement de première ligne des patients atteints de MMND, éligibles à l'autogreffe de cellules souches hématopoiétiques (à l'exception des patients avec un score ISS à 3 et/ou un haut risque cytogénétique), avec un profil de toxicité acceptable. Des essais randomisés prospectifs permettront de mieux étudier cette association qui pourrait prochainement devenir le standard de première ligne dans ce contexte. ]

\section{Références}

[1] Attal M, Lauwers-Cances V, Hulin C, et al. Lenalidomide, Bortezomib, and Dexamethasone with Transplantation for Myeloma. N Engl J Med 2017 ; 376 : 1311-20.

[2] Moreau P, Attal M, Hulin C, et al. Bortezomib, thalidomide, and dexaméthasone with or without daratumumab before and after autologous stemcell transplantation for newly diagnosed multiple myeloma (CASSIOPEIA) : a randomised, open-label, phase 3 study. Lancet 2019 ; 394 : 29-38.

[3] Voorhees PM, Kaufman JL, Laubach J, et al. Daratumumab, lénalidomide, bortézomib, and dexaméthasone for transplant-eligible newly diagnosed multiple myeloma : the GRIFFIN trial. Blood $2020 ; 136: 936-45$ 